\title{
Super-hydrophobic Membrane based on PVDF/Zno Composite Electrospun Nanofibers for wastewater \& Oil spill treatment
}

\section{Azza Mohamed Saad, Ali Sultan Al-Maadeed, AbdulazizMorshidAl-Mohannadi, Dr. Hemalatha, Dr. Jolly Bhadra}

\section{Introduction}

Industrial development releases plenty of oil/dyes wastes to water making unsafe environment to live in. This project aims to solve the problems associated with environmental pollution and discharge industrial effluents to water bodies. This is very important in the global scenario as water is the basis of life. The problem of leaking oil and dyes in water bodies is one of the biggest problems that cause instability in the ecosystem since, it has a negative impact on marine creatures that live in these water bodies. In addition, this damage is transmitted to humans in an indirect way.

The current project aims to develop $\mathrm{ZnO} / \mathrm{PVDF}$ composite nanofibers using electrospun technique. The fabricated nanofibers was analyzed mainly for their morphology by using scanning electron microscope, x-ray diffraction, mechanical tensile strength, UV light spectroscope and oil separation research and photocatalytic performance. Using this nanoparticles oil absorption was done for the water contaminated with oil and dye.
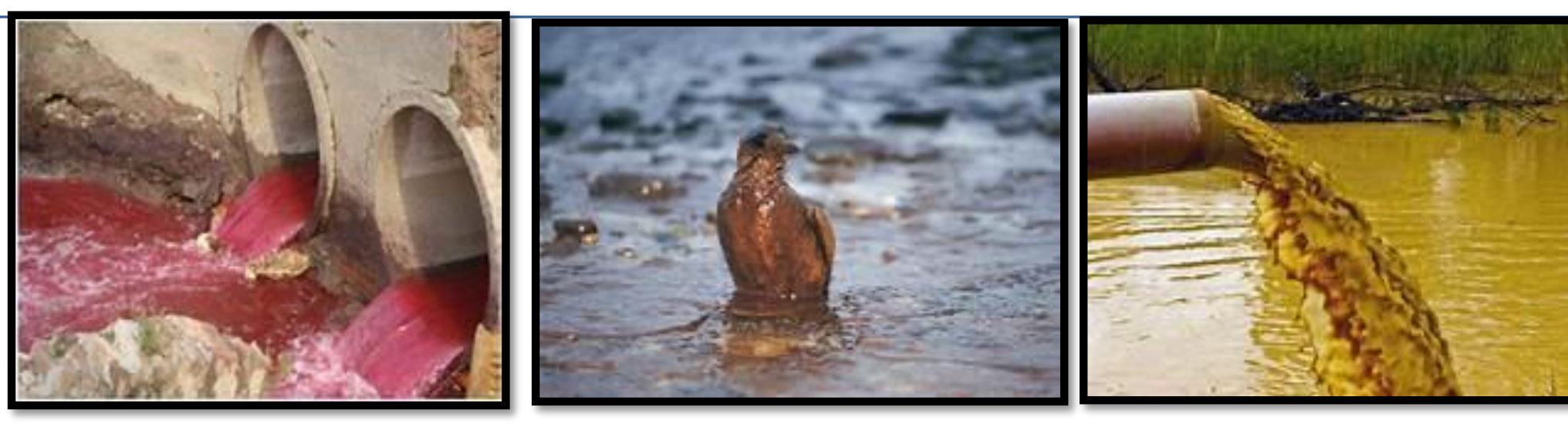

Figure 1: Photos show the Research Problem Research Objectives

To apply the nanotechnology to develop materials for wastewater treatment

* To alleviate environmental pollution and to create a clean atmosphere to live.

- To prepare nanofibers by electrospinning method.

\section{Materials and Equipment}

\begin{tabular}{|l|l|}
\hline Materials & Equipment \\
\hline 1. Zinc Acetate & 1.Beakers \\
\hline $\begin{array}{l}\text { 2.PolyethyleneGlycol } \\
\text { (PEG) }\end{array}$ & $\begin{array}{l}\text { 2.Vacuumpressure } \\
\text { pump }\end{array}$ \\
\hline 3. Ethanolamine & 3. Hot air Oven \\
\hline $\begin{array}{l}\text { 4.Polyvinylidene } \\
\text { fluoride(PVDF) }\end{array}$ & 4.Digi-Drop \\
\hline $\begin{array}{l}\text { 5.Dimethylformamide } \\
\text { (DMF) }\end{array}$ & $\begin{array}{l}\text { 5.Scanning Electron } \\
\text { Microscope (SEM) }\end{array}$ \\
\hline 6.XRD \\
\hline 7. Contact angle \\
\hline 8. magnetic stirring \\
\hline
\end{tabular}

Figure 2 : The photos show the procedure to synthesis of Zinc experiments was mainly targeted for the proposed

\section{Methodology}

\section{PVDF/ZnO composite solutions}

* The first solution was made by adding $0.04 \mathrm{~g}$ of Zinc Oxide to [3ml DMF $+3 \mathrm{ml}$ Acetone] and the second solution was made by $\mathrm{PVDF}+[7 \mathrm{ml} \mathrm{DMF}+7 \mathrm{ml}$ Acetone $]$.

* Then the two solutions were stirred at 70 degrees Celsius for 3 hours. Finally, we mixed the two solutions and stirred them overnight.

3. Fabricated the super-hydrophobic membrane based on PVDF/ZnO composite
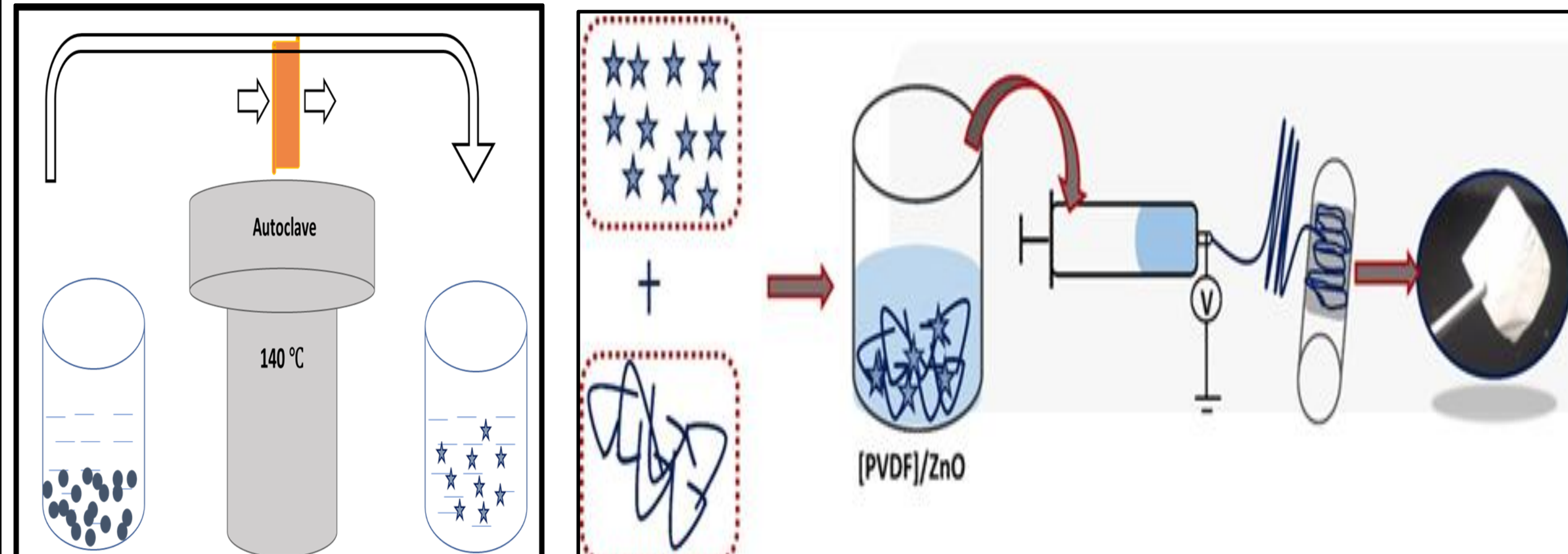

Figure 3: preparation procedure and the electrospinning setup to obtain the PVDF/ZnO composite nanofibers

Experimental Design \& Design tools
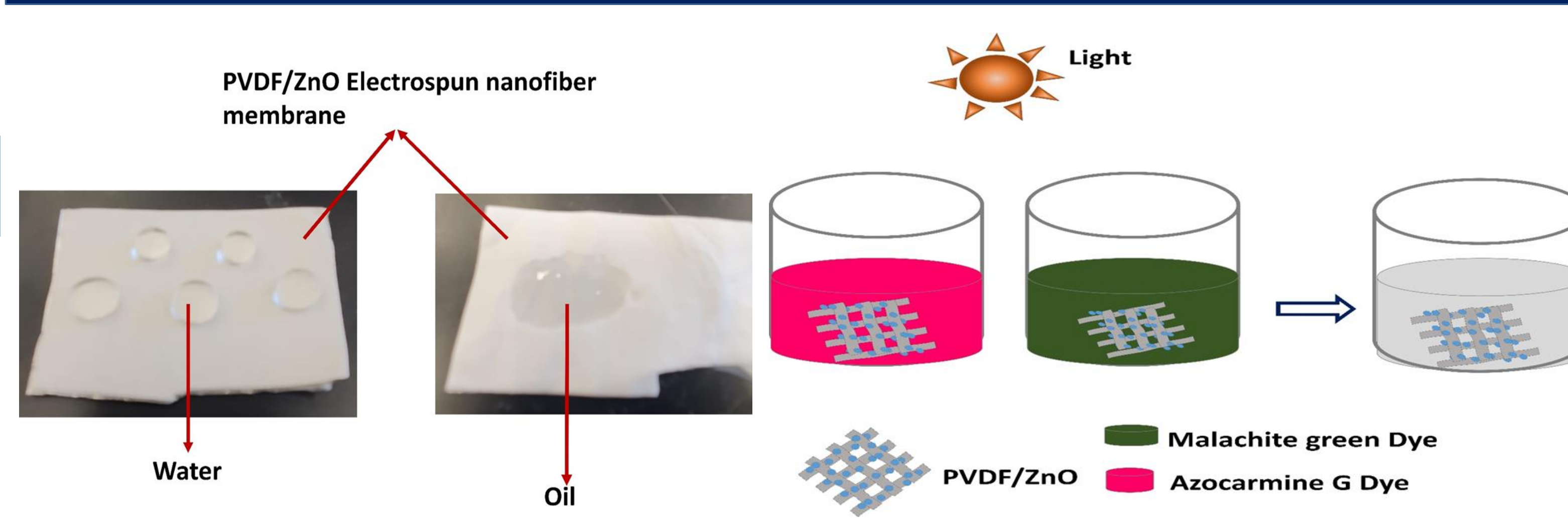

Data and Information Analysis

1. SEM (Scanning Electron Microscope)
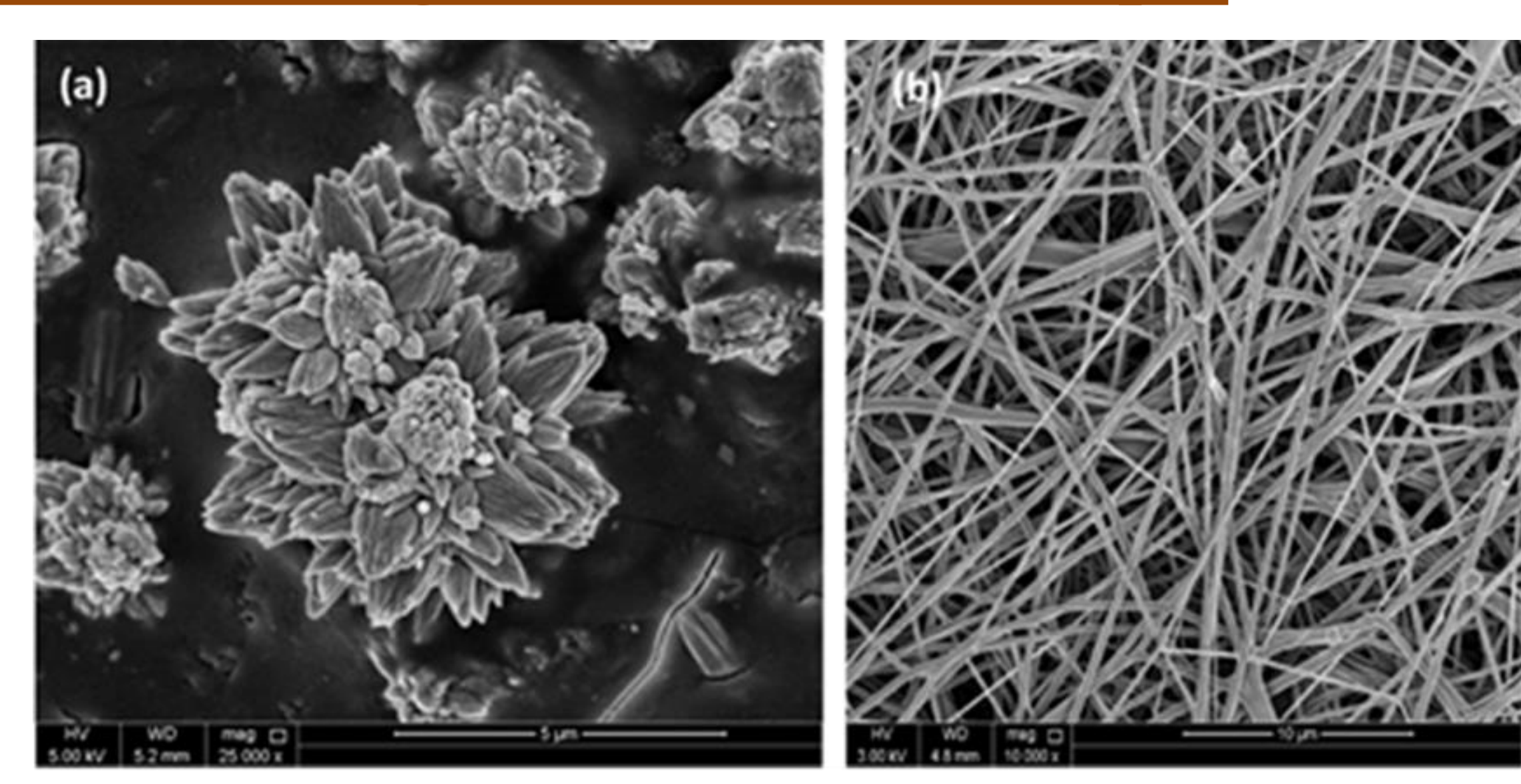

Figure 4: SEM images of (a) pure $\mathrm{ZnO}$ and (b) PVDF/ZnO electrospun nanofiber The SEM results shows The pure $\mathrm{ZnO}$ sample shows the Nano-flower in Figure 4a. SEM image of PVDF/ZnO composite fibers is shown in Figure $4 \mathrm{~b}$ and the average fiber diameter was found to be $70 \mathrm{~nm}$.

It is also seen that all the nanoparticles are dispersed uniformly in the PVDF matrix with no agglomeration.

\section{X-ray diffiraction analysis}

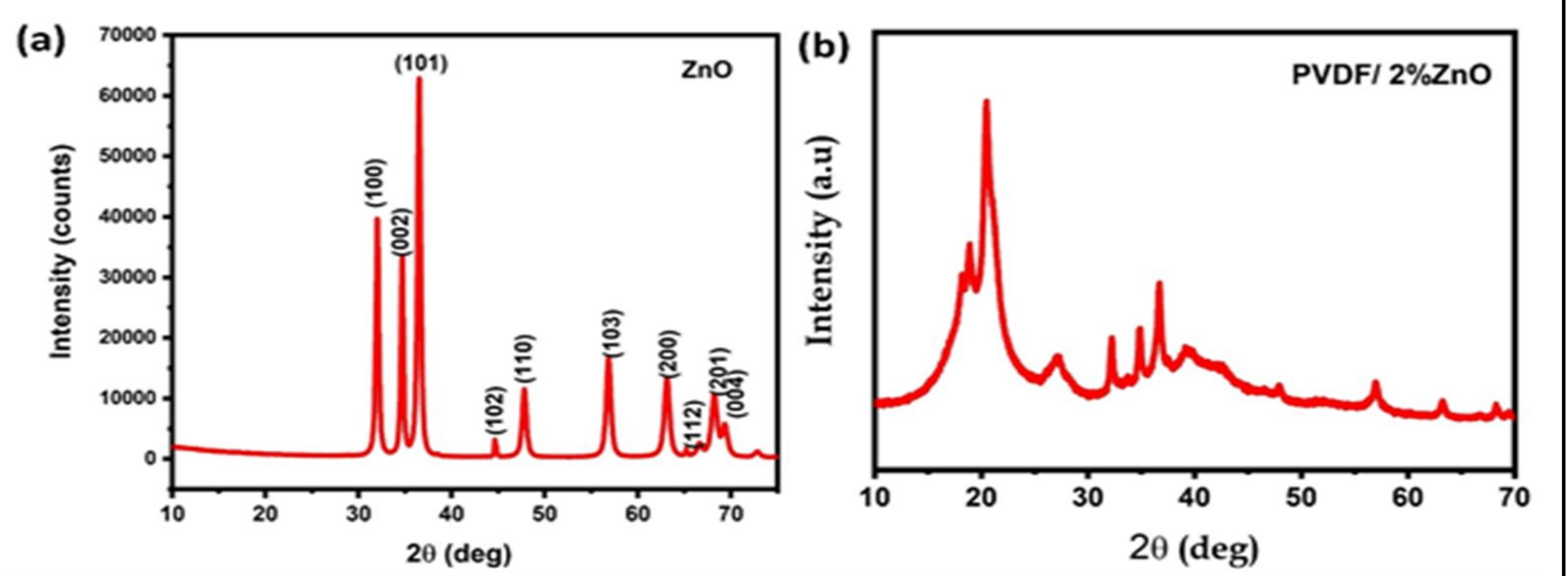

Figure 5: X-ray Diffraction patterns of (a) pure $\mathrm{ZnO}$ and (b) PVDF/ZnO electrospun nanofiber

\section{References}

H. Parangusa, D. Ponnamma, MK Hassan, S. Adham, MAA Al-Madeed, Materials, 12,709, 2019

H. Parangusan, D. Ponnamma, MAA AI-Maadeed, photochemistry and photobiology, 94, 246, 2018.

Q. Ding Y E Miao, T. Li, ACS Appl. Mater. Interfaces 2013, 5, 5617-5622.

D. Ponnamma, S. Nair, H. Parangusa, MK Hassan, S. Adham, A. Karim, MAA Al-Maadeed, Polymers, 12, 4, 2020.

\section{Benefits to Qatar}

Qatar exports natural gas and oil to many countries whose needs are not sufficient from these sources. Therefore, it is possible that the water will be polluted by the effects resulting from the transportation of oil, whether through gas pipelines or via ships. So the composite membrane can be used to adsorb this oil from water, it has a great impact on the environment, and if we can reuse this absorbing oit, it will increase the economy for Qatar In addition, this will conserve the marine creatures, environment and our lives.

\section{Data and Information Analysis}

3) Tensile properties of PVDF/ZnO composite fibers

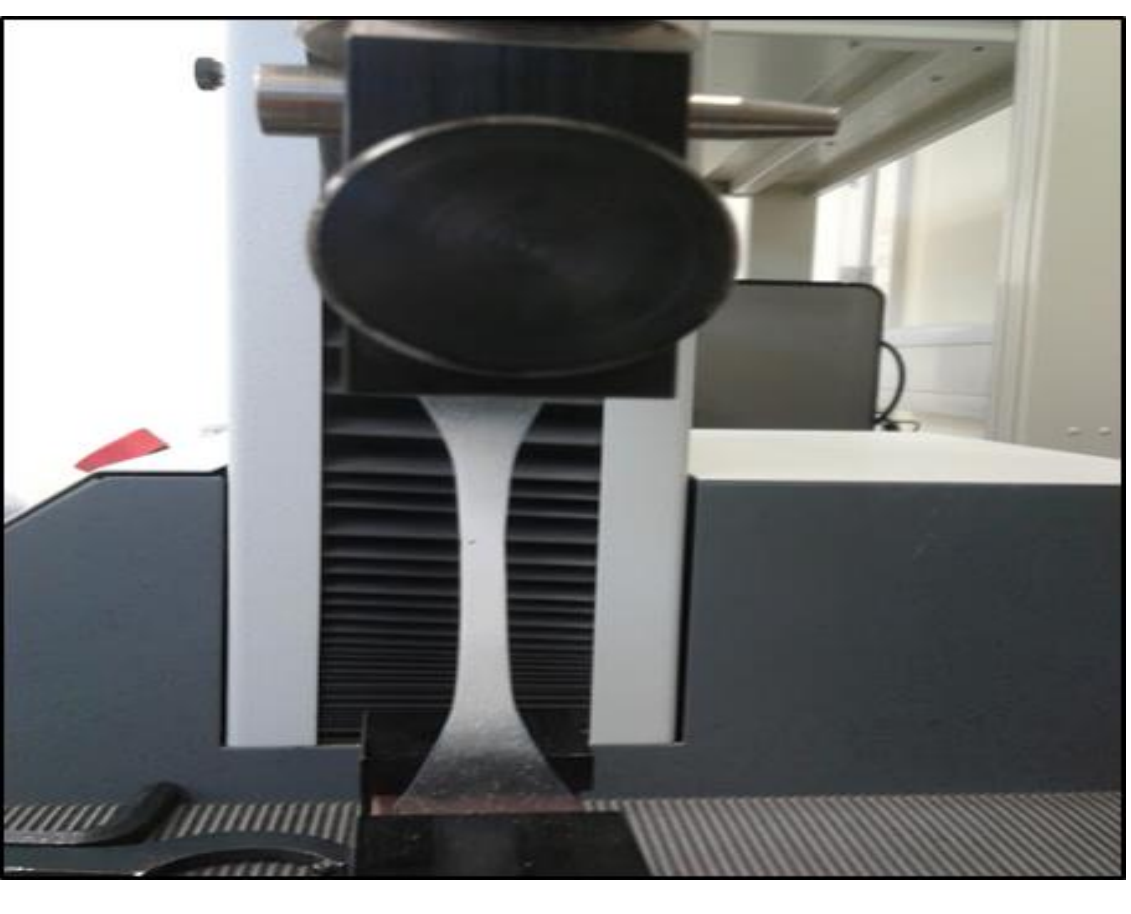

Figure 6: The Lioyd's Universal tensile tester in action

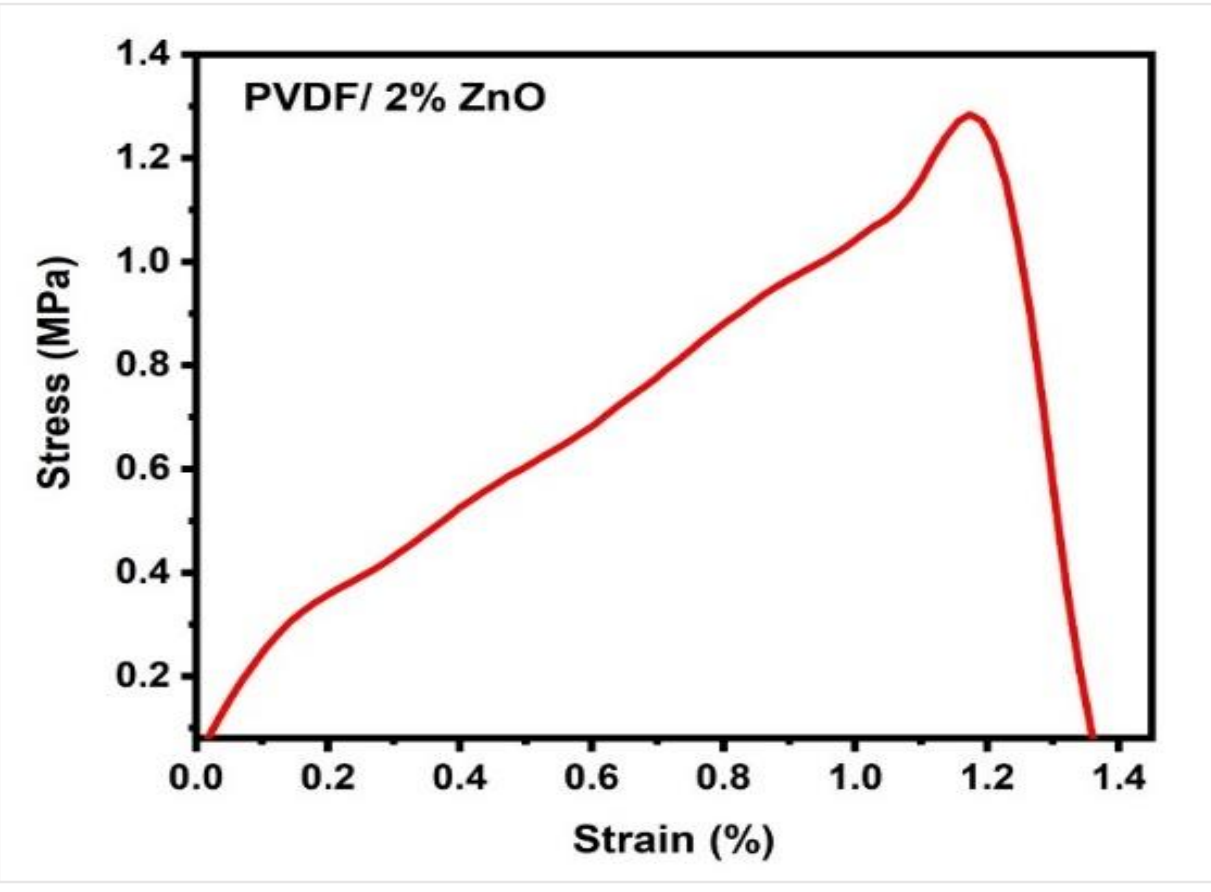

Figure 7: The stress-strain curve of $\mathrm{ZnO} / \mathrm{PVDF}$ composite
The tensile stress for PVDF/ZnO nanofibers was $1.3 \mathrm{MPa}$ it indicates that the mechanical property is higher for the prepared composite fibers.

- The higher mechanical properties obtained for the $\mathrm{PVDF} / \mathrm{ZnO}$ composite fibers is due to the interaction between the PVDF and $\mathrm{ZnO}$ nanostructure

\section{4) Contact Angle Measurements}

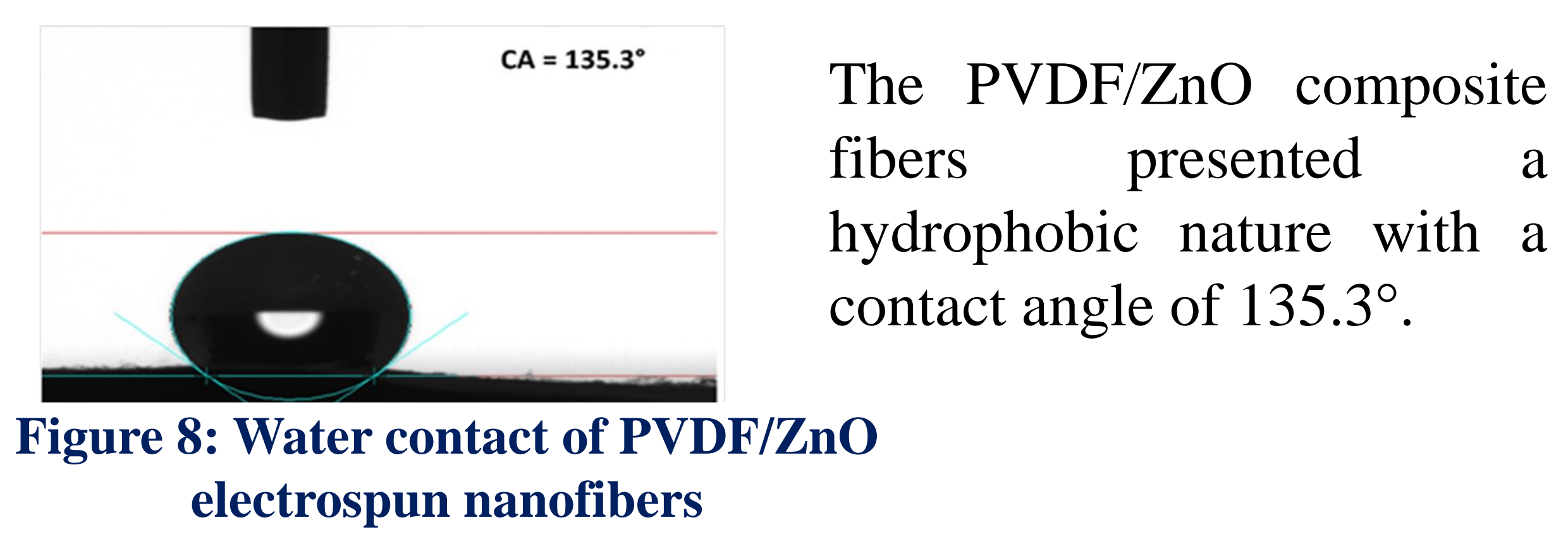

5)Surface wettability of the PVDF/ZnO nanocomposite fibers by Oil and Water

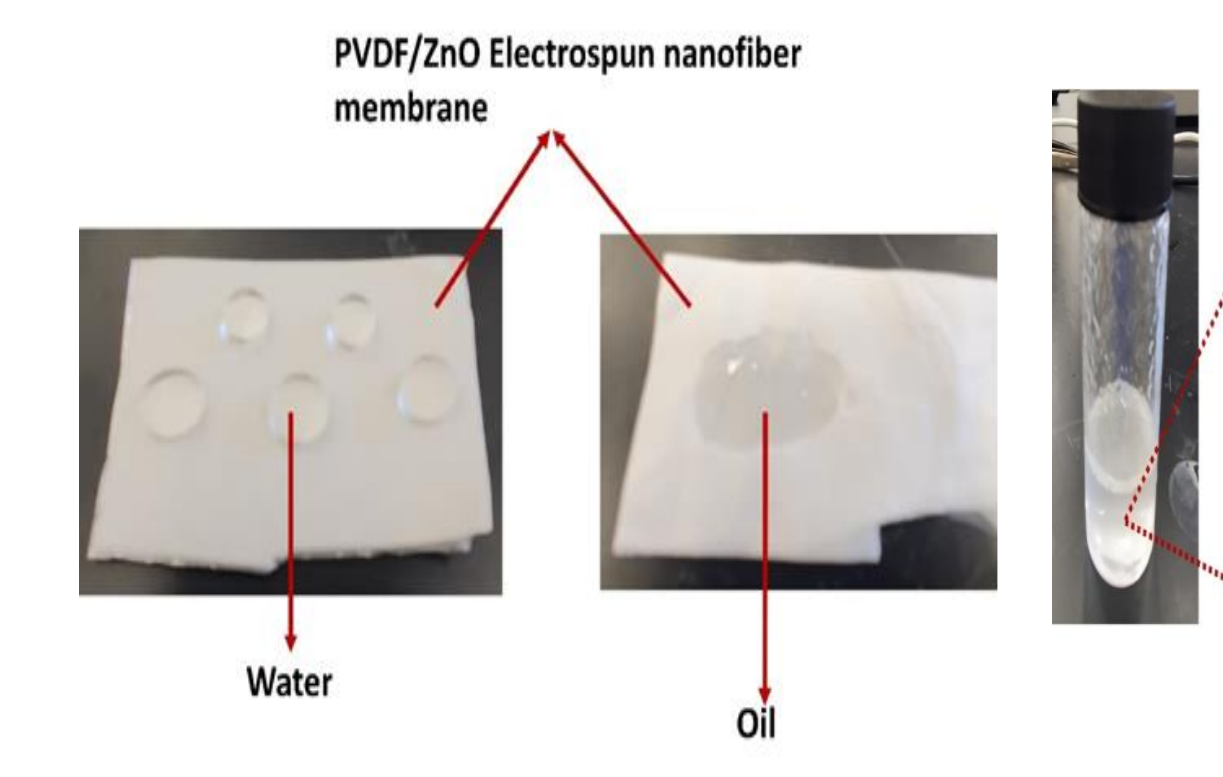

Figure 9: Snapshot showing oil and water droplets on the membranes.

- The surface wettability of the PVDF/ZnO electrospun nanofibers shows that the fabricated composites hydrophobicity while the water droplets rest on the fiber surface and oil particles penetrate through the fibers.

* The optical images shows that no oil presences after the absorption.

\section{6) Dye Removal}
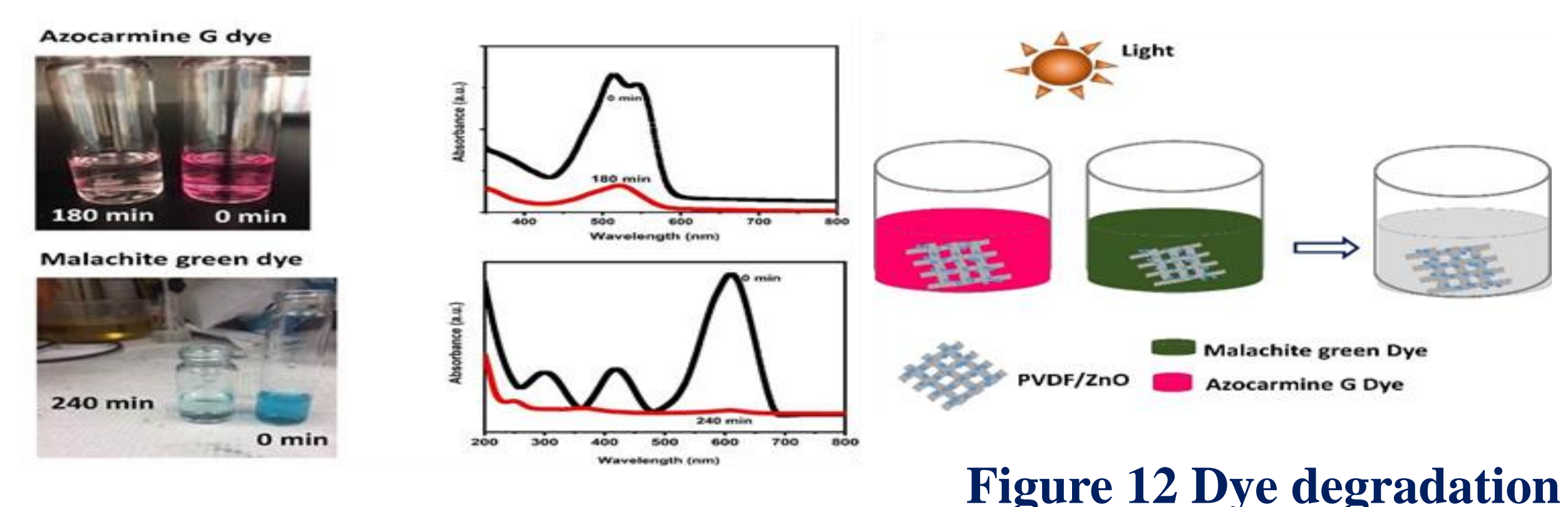

Figure 11: Absorption spectrum of

(a) Azocarmine G and (b) Malachite

Figure 12 Dye degradation

The absorption spectrum of dye solution indicates that the concentration of dyes in the solution decreases rapidly and virtually vanishes in $180 \mathrm{~min}$ (Azocarmine $\mathrm{G}$ ) and $240 \mathrm{~min}$ (Malachite green)

\section{Results}

In summary, we have successfully synthesized the flower-like $\mathrm{ZnO}$ architectures by hydrothermal method. Developing oil and dye absorbing membrane using electrospinning techniques. It can absorb oil and dyes from water wastes, can be reused many times. The observed results suggest that the fabricated flexible electrospun nanofibers is suitable for the wastewater treatment.

\section{Recommendations}

1. Testing different types of dyes not mentioned in this. . Mix two dyes together and check testing by the fabricated membrane.

Testing mixture of both oil and dye wastes together. 4. Development the membrane to be as sponge can hold the oil then collecting this oil and reuse it again. 